\title{
EDITORIAL
}

\section{Why is the Acute Watery Diarrhea in Ethiopia Attaining Extended Course?}

\author{
Abraham Haileamlak, MD, Professor of Pediatrics and Child Health \\ DOI: http://dx.doi.org/10.4314/ejhs.v26i5.1
}

Acute Watery Diarrhea (AWD) is an acute illness which has remained as one of the important public health problems in Asia and Africa causing substantial morbidity and mortality. It occurs both as a short outbreak and protracted epidemic/pandemic. The transmission of the causative bacteria is usually through the fecaloral route of contaminated food or water caused by poor sanitation.

From late 2014 to end of 2015, AWD outbreak has grasped 30 of the 47 counties of Kenya causing close to 7000 cases of morbidity and over 100 deaths. The outbreak occurred in light of the El Niño floods which created favorable conditions for the spread of the causative bacteria. After spreading in Kenya for over a year, the disease moved to Ethiopia.

In Ethiopia, AWD was first reported in districts of Oromia and Ethiopian Somali close to the Ethio-Kenyan border in February 2016. Thereafter, it continued spreading east and north reaching many parts of the country including the capital city and the northern parts of the country. Despite an ongoing effort to contain the outbreak, it continued spreading to large areas of the country affecting a large number of people; its course remained protracted and kept many people at risk.

The major contributors for the occurrence of such outbreak and the propagated dissemination of the disease are:

Climatic condition: Most often AWD outbreak occurs in humid and intertropical zones during rainy seasons. Flooding during rainy seasons can favor the emergence or the upsurge of AWD outbreak. Although the causative bacteria are more fragile in the atmospheric environment than in its aquatic environment, it can survive well for some days on fruits and vegetables. Ecosystem changes due to global warming, environmental pollution, drought, deforestation leading to inundations and other climatic disasters are the reason for the upsurge of AWD outbreak. The consecutive severe droughts in the previous years followed by excessive rain and flooding in Eastern African countries is typical of the above features which favor for such outbreak.

Demographic and social factors: Demographic explosion especially in big citiesassociated with poverty and chaotic urbanization is a key factor in the occurrence of the outbreak. Big gatherings (pilgrimages and celebrations) that favor overcrowding and lack of hygiene are usual and very well known risk factors for the occurrence of such an outbreak.

On top of the above prevailing conditions, the other elements contributing to the increase of the spread of the causative organism in the population at risk are: insufficient WASH access, population density, poor awareness about the disease and way of life.

The current issue (Vol. 26, No 5), the fifth issue of the year, contains one editorial focusing on current issue-Acute Watery Diarrhea, nine original articles on varied subjects and four case reports dealing with different topics. I invite readers to read through these articles and appreciate or utilize the contents. I also encourage readers to forward comments and suggestions. 